Tubular Adenocarcinoma.-The tumour of the secreting element is the tubular adenocarcinoma. It is much more malignant, rapidly infiltrating the parenchyma in which it arises. It involves the perinephric tissue early, and produced metastases in the adjacent lymphatic glands. It thus comes about that the kidney, although increased in size, may have a normal contour and the drainage system be only slightly damaged, so that pyelography reveals little deformity, the cavity appearing probably dilated and at one part slightly deformed. When examined by excretion urography, however, it is demonstrated to be a functionless organ by the absence of any shadow. A rare tumour of the renal parenchyma is a haemangioblastoma.

Papillary Adenocarcinoma.-The commonest malignant tumour arising from the collecting element is the papillary adenocarcinoma. Many of these were formerly mistaken for hypernephromata, and, like the latter, they formed a tumour mass, usually palpable and demonstrable. The characteristic local destruction of the drainage system, with the neighbouring calyces elongated and flattened, is observed on pyelographic examination. Functional activity as estimated by excretion urography, however, is poor. From the pelvis or calyces an epidermoid carcinoma may arise.

Villous Papilloma.-The innocent tumour of the kidney that is met with clinically is a villous papilloma. It is similar to that which occurs in the bladder. Its presence can be recognized by pyelography. At first sight, when the film is inspected, the pelvis and calyces appear normal, but on more careful examination the density of the media within the cavity is seen at one part to be diminished-namely, where the villous growth is occupying the cavity. In some cases a definite filling defect is observed, and when this is combined with an interference with the entrance of the pyelographic media to one of the calyces it may be suspected that the villous growth has overstepped the borderline and become a malignant papilloma. Functional activity as estimated by excretion urography in these cases is good.

Tuberculous disease of the kidney produces various appearances on pyelographic examination, which are characteristic and diagnostic.

Renal Calculus.-If the shadow of a stone is seen in the renal region at the preliminary $x$-ray examination, its accurate localization can be simply achieved by taking another photograph with the patient lying on his side. If the concretion be situated within the renal pelvis, th:s lateral view will show it lying opposite the anterior third of the body of the corresponding lumbar vertebra. If the shadow be intra-abdominal, it will be seen in front of the bodies of the lumbar vertebrae. The exact situation of the stone within the pelvis or calyces can be demonstrated by pyelographic examination.

\section{IDIOPATHIC HaEmaturia}

Essential or idiopathic haematuria is the one renal disease producing haematuria whose diagnosis is arrived at by a process of exclusion. The source of the bleeding having been localized to one kidney, no further abnormality of this organ can be revealed by any of the various methods that are employed; the urine collected is found to be sterile and free from pus cells, such as would be met with if the bleeding was from an inflamed pelvis; the pyelographic contour is normal. In some cases of essential haematuria the bleeding may be most persistent. Eight years ago I operated on a man who had suffered from haematuria from this cause for twenty out of twenty-one years since it originally appeared. Nephrectomy was performed, and he has had no recurrence of the bleeding since then. Anatomically, after the kidney was removed it appeared normal, and on histological examination all that was revealed was a certain increase of the intrarenal pelvic fat, and a varicosity of the venules within it.

\section{Conclusion}

When a case of haematuria comes under our observation it is our duty to demand that the cause of the bleeding be forthwith determined. This may necessitate a somewhat tedious examination, but the ultimate result is certain. Fortunately, in most cases, when the cause of the bleeding has been determined, a speedy and certain cure can be obtained. In others, especially where malignant disease is present, the outlook is graver. In all of them, however, the earlier the diagnosis is arrived at the better will be the result of treatment, and in all of them, whether they are innocent or malignant, the progress of the case afterwards should be followed up by re-examination at regular intervals of three months, six months, and a year. It is difficult to persuade patients who have been pronounced cured after operative treatment of the necessity of this, and the temptation sometimes is to dismiss them with a reassurance that all is well for all time. Fortunately, in many this will prove to be correct, but in others, slight recurrence may take place, and, if so, by an accurate follow-up system such recurrence can be observed at its earliest stage and dealt with simply and promptly.

\section{THE STROKE IN HIGH ARTERIAL PRESSURE* A STUDY OF 150 CASES \\ BY}

H. O. GUNEWARDENE, M.B.,, B.S.LoNd. D.M.R.E.CANTAB,

FORMERLY CLINICAL ASSISTANT, NATIONAL HOSPITAL FOR DISEASES OF THE HEART, LONDON

The prominence given to the study of high arterial blood pressure seems to be ever increasing, both in the recognized textbooks and in the medical journals. Since Clifford Allbutt's lucid exposition of the conditicn called by him " hyperpiesis," Batty Shaw, Geoffrey Evans, Lord Dawson, and others have contributed to the elucidation of some of the clinical and pathological aspects of this type of high arterial pressure and of that associated with renal disease. But much remains still hazy, unknown, and unexplained. Etiology is but vaguely understood; some of the clinical manifestations have received no uniform explanation, physiologically or pathologically ; and the lines of treatment indicated touch no specific methods which can be relied on to help the practitioner, who is left to turn for relief to general measures adopted in the treatment of other diseases-for example, rest, restricted diet, hydrotherapy, and the removal of septic foci.

On the whole, one feels that there is some justification for striking a note of disappointment, and that, in spite of the valuable contributions hitherto made, very little substantial advance has taken place in our knowledge by which the disease can be arrested or relief be given to those in danger of being afflicted with its unpleasant sequels. One reason for this slow progress seems to be clear-namely, that the malady has not been thoroughly investigated or its early stages closely followed. Cerebral haemorrhage and heart failure as terminal events in high blood pressure may be encountered commonly enough in hospital practice, but the earlier phases and the less serious and striking manifestations are very seldom seen. Indeed, it is true to say that, by the time the grosser

* Awarded Sir Charles Hastings Clinical Prize, 1930. 
lesions have developed, the patient has already ceased or forgotten to look upon the earlier symptoms as part of the same disease in its undeveloped state.

High blood pressure, whatever its etiology, is common enough in general practice. No one is in a better position to study those aspects of it which throw light on its causation, and on some of the features which forecast the final doom, than the family practitioner. Methods of investigation are no doubt limited, but the mass of clinical material is heavy. No apology is made for not resorting to those special tests which can be carried out only in a fully equipped laboratory with a full staff, for the purpose of this essay is to record the clinical observations made on the so-called "stroke" as it occurs in high arterial pressure.

\section{Definition of "STROKe"}

A stroke is defined in Dorland's Medical Dictionary as " a sudden severe attack, as of apoplexy or paralysis." Saville considers "stroke" to be synonymous with apoplexy, for he defines each word as " a term which may be conveniently retained to indicate a sudden unconsciousness due to a vascular lesion within the skull." I propose to resort to a certain degree of licence in the use of the word, interpreting it as a " sudden severe attack," not necessarily apoplectic, and thus implying that these attacks are not confined only to the motor side. There appears to be some justification for such a departure, for one finds Batty Shaw saying (under the heading "Persistent or temporary hyperpiesic paralysis") : "Sometimes the paralysis affects the sensory instead of motor areas, so that the patient becomes blinded without any changes being discovered in the retina or the brain." A little elasticity in my definition seems justified, not only because it helps to explain more fully the various forms these attacks take, particularly in the earlier stages of high blood pressure, but also because it helps to give a mental picture of the much-neglected sensory forms.

\section{FreQuency of STROKe}

Stroke occurs so commonly in high pressure, at least in Ceylon, that the scant references to it in standard textbooks is surprising. Theodore Thomson in Price's Textbook of Medicine says, " sometimes transient paralysis occurs," without any mention of the sensory attacks. Batty Shaw makes the statement quoted above in a superficial but none too impressive way. East and Bain, in Recent Advances in Cardiology, make no reference to the sensory stroke. In what they call the second stage, they say, "symptoms will rarely be experienced which refer to the pressure," also stating that the clinical features "are strikingly few "- - this is in contrast to my finding that the strokes, all except the apoplectic, are most common at this stage. Vaquez, on the other hand, states clearly: "Transient aphasia and fleeting paresis, specially brachial monoplegia, are frequent incidents in vascular hypertension. They appear and disappear suddenly, leaving no traces, and may occur several months later." He lays some stress on the mental symptoms (uncommon in my series), which, he maintains, assume the most varied forms-for example, amnesia, mental confusion, and suicidal tendency.

\section{Motor and Sensory Strokes}

Defining "the stroke" as a sudden severe attack drawing the attention of the subject of hypertension to a change in his normal motor or sensory phenomena, one can classify " motor strokes" under the main heads of the paralytic and the paretic. These can be described according to distribution as: (1) hemiplegic ; (2) mono- plegic ; (3) localized-for example, tongue ; and (4) diffuse. The last variety usually occurs as a terminal event, being due to a severe haemorrhage which causes sudden death, or to one which produces paralysis, first of one side of the body and then of the other. According to their duration, we may say that they are: (1) permanent, (2) present for a few days or weeks, and (3) transient. The annexed diagram gives a clear picture of what may occur on the motor side.

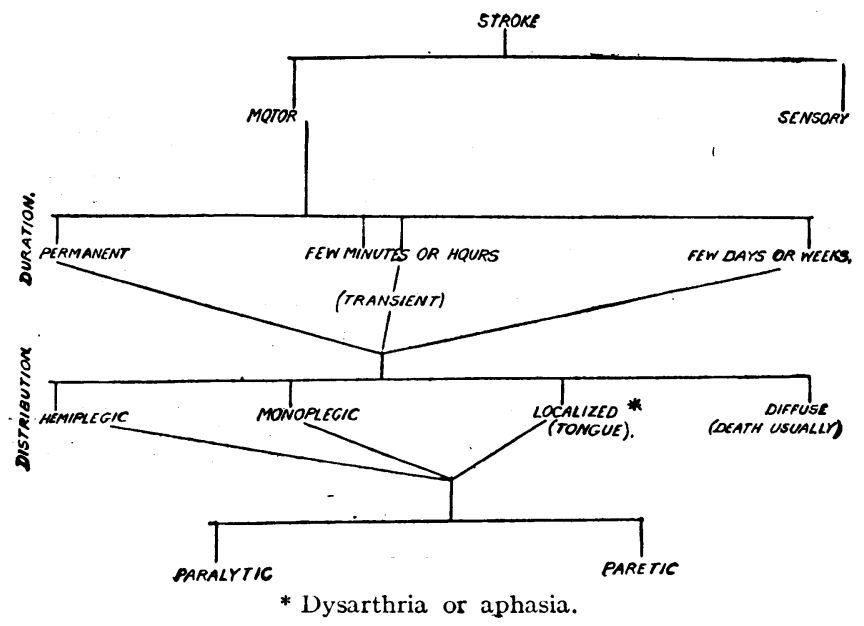

On the sensory side we get similar changes: complete loss of sensation, as in coma, and changes in sensationnamed " incomplete" for the purpose of the diagramthese being analogous to the paralytic and the paretic forms on the motor side. The distribution and duration are similar to those occurring in the motor phenomena. The diffuse form, including cases in which tingling or numbness, or " a peculiar feeling all over the head and body," has been reported, is rare. Changes in sensation have been variously described by the patients themselves, and may occur associated with, in the absence of, or following motor phenomena. Tingling and numbness are

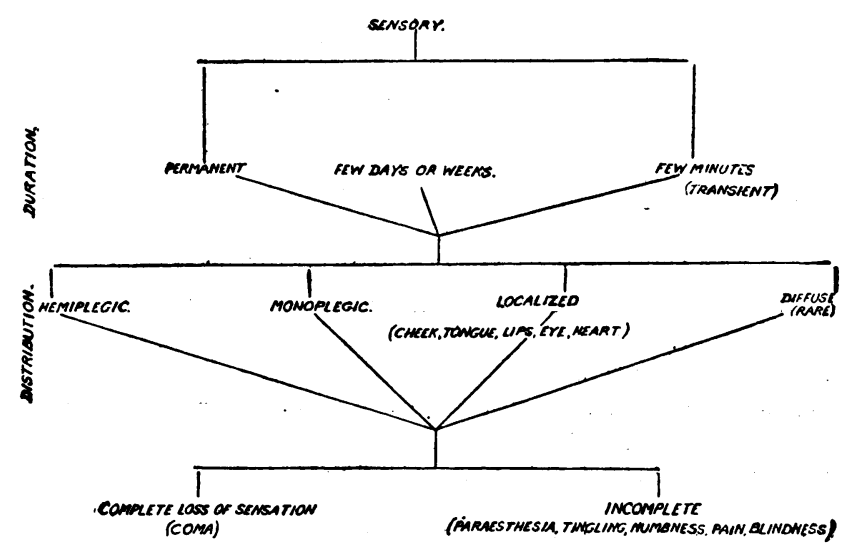

common. One patient of mine described the sensation as " a cotton-woolly feeling-the face feels heavy on that side." A doctor, in whom a monoplegia had disappeared, described as irritating " the peculiar dry feeling of the limb, as though it had been immersed in formalin and taken out" ; again, "When warm water is taken I feel it hotter on one side of the tongue; and, further, on shaving I do not feel the razor well on the recently affected side." In another case a patient complained of numbness of the ear, left upper limb, and distal part of the left leg after motor power had returned His description was: " as though I have placed my hand on a lump of ice and removed it." Of the ear he said: "When I scratch my ear it feels plain." 


\section{Diastolic Pressure in Stroke}

It is interesting to study very clearly at what stages in high arterial pressure the stroke supervenes. In my series of cases the stroke, sensory or motor, has not appeared in a single instance in which the diastolic blood pressure was under 115 . In 20 per cent. of the cases the stroke-in most transient, in a few permanent, in one fatal-occurred with a diastolic blood pressure between 115 and 120 . In ten out of the 150 cases death resulted from what appeared clinically to be definite cerebral haemorrhage ; in nine of these, the patients were actively or restrictedly pursuing their usual occupations. Again, in nine of these ten the diastolic blood pressure was over 135, the systolic being 200 and over. In 10 per cent. of the series the transient and permanent strokes occurred in cases with a diastolic blood pressure of over 125 .

It seems, therefore, that sudden changes in the normal sensory and motor phenomena appear most commonly with a diastolic blood pressure between 115 and 125, and are altogether absent in cases with diastolic blood pressures under 115. Cerebral haemorrhage seems to be uncommon in patients under observation, and they are warned against any but a very quiet life. Fatal haemorrhage seems to ensue mostly in patients who carry on ordinary occupations without making allowances for their abnormal pressure. Indeed, it seems that the catastrophe of fatal haemorrhage is the one phase of high arterial pressure in which the practitioner or specialist can give little relief. There is therefore all the more urgent need to study the earlier signs and symptoms very fully, for then therapeutic measures can be resorted to in time, and where these are likely to be of no avail, relatives and dependants may be warned of the possibilities ahead, and adjustments relating to business, finance, or family matters may be made.

\section{Heart Failure and Stroke}

The relation of the stroke to heart failure consequent on high arterial pressure is worth studying. My observations seem to suggest that fatal cerebral haemorrhage, or even transient strokes, are very uncommon once failure has set in or the heart has begun to show considerable enlargement, with dyspnoea and other symptoms of a tiring cardiac musculature-even though at this stage it is observed that the blood pressure, systolic and diastolic, remains high. In three cases of mine, however, there was very marked enlargement of the heart with the apex in the sixth space and anterior axillary line, with thickened vessels and a locomotor pulse (brachial), but with practically no symptoms referable to the heart. These three patients are reported to have died of cerebral haemorrhage. The impression is gained, in a review of my cases, that patients with hyperpiesis, showing few or no symptoms referable to the heart, with or without cardiac enlargement, are particularly prone to die of cerebral haemorrhage.

\section{Diagnosis and Prognosis}

In this country (Ceylon) there is a popular belief that the native doctor, so called because he practises Ayurvedic medicine, excels the practitioner of Western medicine in the diagnosis and treatment of paralysis. This is no doubt due, in my opinion, to our mistakes in the matter of prognosis in these cases, and to our failure to appreciate the transient nature of the attacks.

A common procedure is as follows. A man gets hemiplegia. The nearest practitioner is sent for ; he diagnoses cerebral haemorrhage and gives a gloomy prognosis, hinting at the possibility or probability of death within twenty-four hours. The blood pressure is not taken.
In despair the relatives run to the native doctor, to whom no circumstances are hopeless, and who not only promises relief, but paints the picture of a permanent cure. The case falls into the hands of the latter, and time, with restricted diet on the one hand and rest on the other, works the miracle. In one case the patient gives this version: "Dr. X saw me, and said it was a ' spasmodic' stroke. We took native treatment, as Dr. X said recovery would take three months, and it was due to high pressure." If the possibility of recovery in a few hours or days had been mentioned with confidence the practitioner would have retained his patient as well as his fees.

In sensory cases the phenomena are generally classed under neurasthenia, neuritis, and hysteria, and the possibility of pressure as a causative factor is missed. Thus in this country, and elsewhere too, misdiagnosis must be common.

These observations have been made with one object in view-namelys to invite the study of this common disease, and so to correct or amplify the present findings. From the point of view of the practitioner there is everything to gain and nothing to lose by agreeing with Cabot that " the measurement of blood pressure is the most important of all the recourses that have been added to our armamentarium in the last fifteen years." When the frequent occurrence of sudden motor and sensory changes as a result of high arterial pressure is more widely recognized, the diagnosis and prognosis will be placed on more reliable data.

\section{Conclusions and Summary}

In a final review of these observations on the stroke in high arterial pressure, one appears entitled to infer:

1. That transient or permanent paralyses of varied distribution occur fairly frequently.

2. That the hitherto neglected sensory phenomena have a distribution similar to the motor phenomena ; that they also occur frequently, and are probably caused through an affection of the sensory areas, the pathogenesis being similar to that in the motor cases.

3. That strokes often attributed to other factors, such as shock, exertion, and emotion, are the result of an already existing pressure exacerbated by the influence of these factors.

4. That cerebral haemorrhage does not seem to occur with diastolic blood pressures of under 115 (whatever may be the systolic), and that prognosis may be based on this observation.

5. That cerebral haemorrhage occurs most commonly in people working in defiance of an already existing pressure, and without taking any account of this abnormality.

6. That any kind of paresis or paralysis is very rare in cases in which the diastolic blood pressure is under 115 . If they do occur, the minimum diastolic pressure is over 115 at the time of the stroke. Strokes at this pressure are rarely fatal or permanent. The former occurs in patients with thickened vessels or other disease; the latter in an unfortunate few, or in those in whom the causative factor is other than hypertension.

7. That cerebral haemorrhage seems to occur more frequently in those cases of hypertension which show neither marked cardiac enlargement nor symptoms, and rarely in those cases in which there are signs both of considerable cardiac enlargement and symptoms of heart failure.

I am grateful to my medical colleagues at the General Hospital, Colombo, for giving me opportunities for verifying my observations with cases in their wards. 